\title{
Low Grade Myofibroblastic Sarcoma in Pre-Existing Fibroadenoma - A Rare Case Report
}

\author{
Dr. Abhishek Verma ${ }^{1}$, Dr. Ravi Murmu ${ }^{2}$, Dr. Somya Sinha ${ }^{3}$, Dr. Anu Singh ${ }^{4}$ \\ ${ }^{I}$ Senior Resident, Department Of Laboratory Medicine, RIMS, Ranchi, India \\ ${ }^{2}$ Junior Resident, Department Of Pathology, RIMS, Ranchi, India \\ ${ }^{3}$ Junior Resident, Department Of Obstetrics \& Gynaecology, Seth GS Medical College, India \\ ${ }^{4}$ Junior Resident, Department Of Pathology, RIMS, Ranchi, India
}

\begin{abstract}
Background: Low grade myofibroblastic sarcoma of breast are rare tumors of myofibroblasts that arise from a pre-existing fibroadenoma or vascular pericytes.

Case Presentation - A 22 year old married woman presented with a slow growing right breast lump which on mammography mimicked galactocele but on histopathological examination revealed to be a low grade myofibroblastic sarcoma arising from a pre-existing fibroadenoma.

Conclusion: The tumor was diagnosed as low grade myofibroblastic sarcoma.

Keywords: Fibroadenoma, Low grade myofibroblastic sarcoma, Smooth muscle Actin

\section{Introduction}

Low grade myofibroblastic sarcoma is a rare mesenchymal tumor that rarely metastasize. It arises from head and neck region but can also arise from retroperitoneum. Cases of myofibroblastic sarcoma arising from breast is extremely rare and few cases have been documented worldwide. Mammary myofibroblastic sarcomas have always been reported as a low-grade rather than a high one. The tumor resembles nodular fascites and myofibroblastoma to fibrosarcoma, leiomyosarcoma and cystosarcoma phylloides. However the cell of origin of myofibroblasts is unclear in all these cases.
\end{abstract}

\section{Case history}

A 22 year old married woman initially presented with a lump in the upper outer quadrant of left breast gradually increasing in size. Mammography done initially gave an impression of a galactocele. The clinical scenario and FNAC, pointed out the diagnosis of giant fibroadenoma and excision was suggested. Lumpectomy was performed. Grossly the mass was 12 x 10 x $5 \mathrm{~cm}$, well circumscribed . The cut section showed homogenous grey white areas. The sections taken were subjected to routine haematoxylin and eosin staining. Various section showed fascicles of spindle cells were seen which had fusiform nuclei with pleomorphism. The cytoplasm was eosinophic with indistinct cytoplasmic borders. The background stroma was variably collagenous ranging from loose collagen in hypercellular areas to dense collagen in hypocellular areas. The periphery of tumour showed intracanalicular pattern of fibroadenoma. Hence, this represented ancient fibroadenoma that was a precursor lesion for low grade myofibroblastic sarcoma. The diagnosis of low grade myofibroblastic sarcoma was offered . which was further confirmed by immunohistochemistry. The tumour cels showed diffuse positivity for smooth muscle actin (SMA)

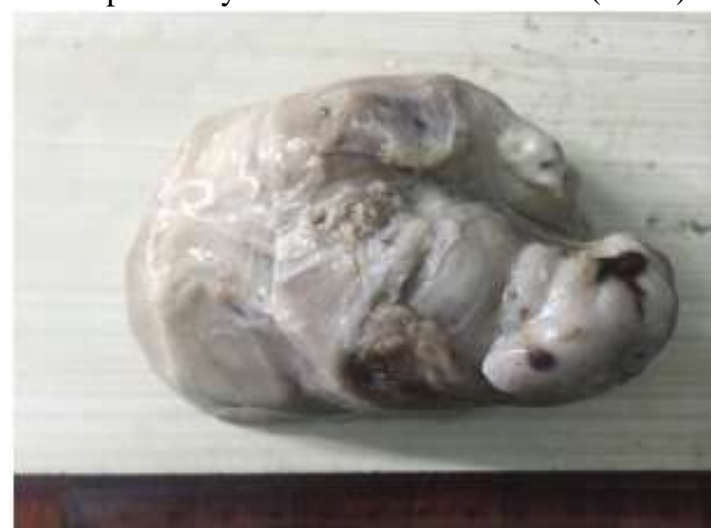

Figure 1. Gross picture of low grade myofibroblastc fibrosarcoma showing multinodularity.

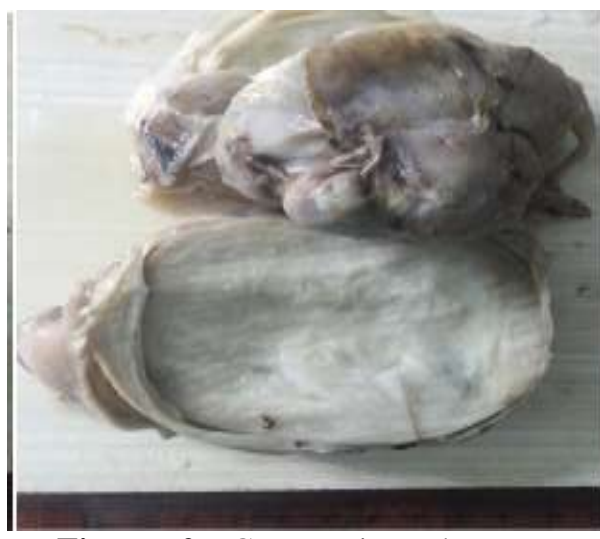

Figure 2. Cut section shows grey white encapsulated tumor. 


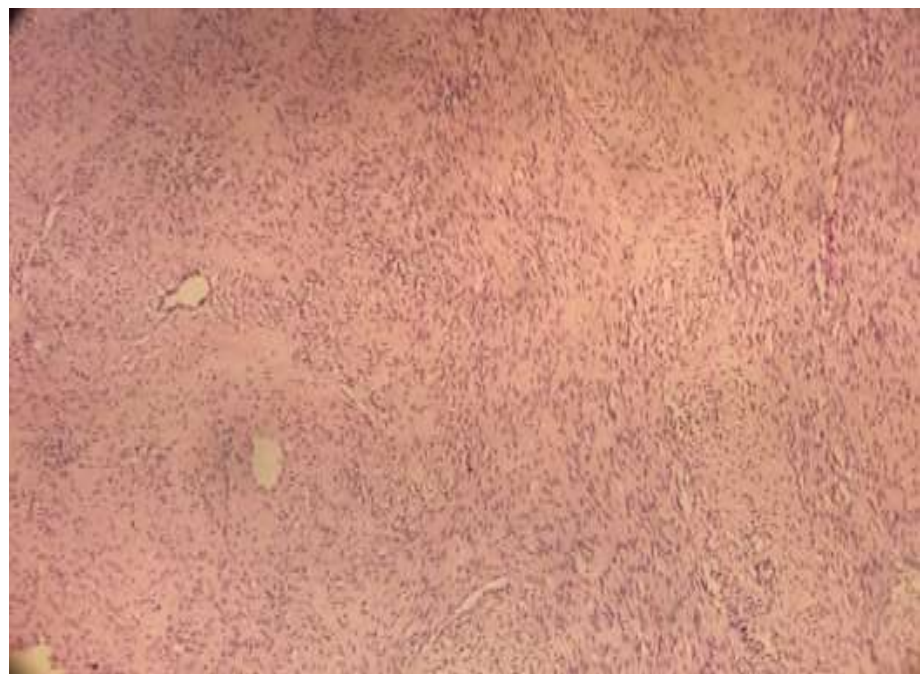

Figure $3-\mathrm{H}$ \& E section of low grade myofibroblastic sarcoma showing plump spindle cells with vascular spaces. (10x view)
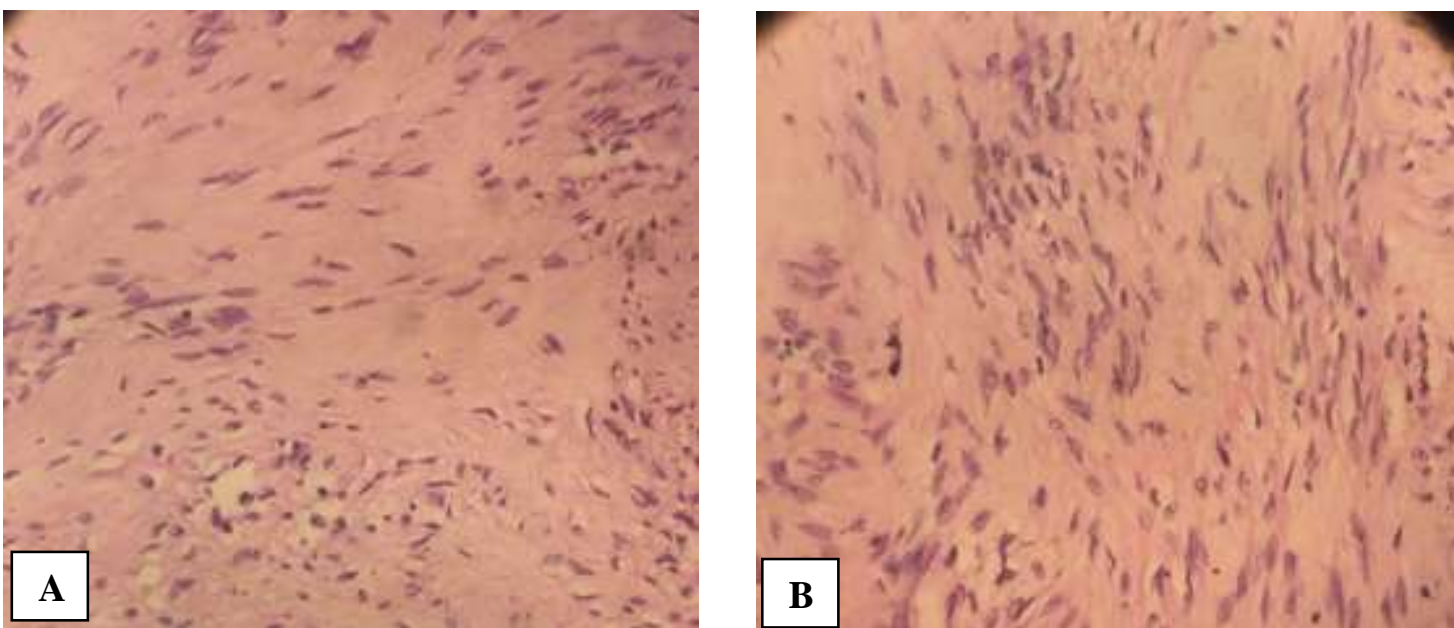

Figure 4 - (A \& B) H \& E section of low grade myofibroblastic sarcoma showing spindle cells intersped between collagen fibres. There are no areas of necrosis. (40x view)

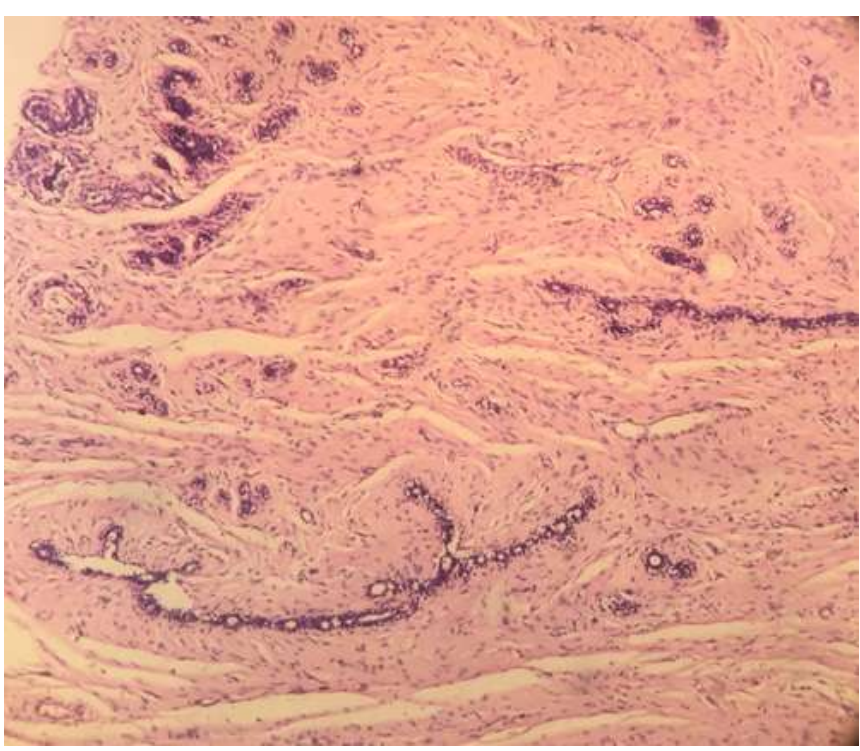

Figure 5 - H \& E section of low grade myofibroblastic sarcoma arising from pre-existing fibroadenoma. (10x view) 


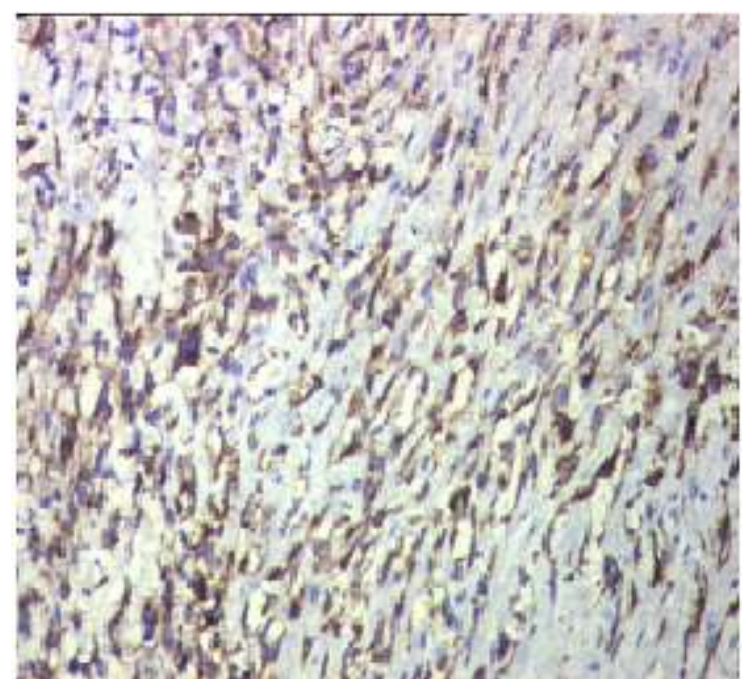

Figure 6 - IHC showing Smooth Muscle Actin (SMA) positivity in low grade myofibroblastic sarcoma breast.

\section{Discussion}

Low grade myofibroblastic sarcomas of the breast can develop from a sarcomatous transformation of long standing fibroadenoma or from vascular pericytes. They are considered as tumors of low malignant potential that are slow growing, locally infiltrative, may recur after local excision but rarely metastasize. They should be distinguished from other similar conditions like pleomorphic sarcoma, fibrosarcoma, leiomyosarcoma and cystosarcoma phylloides. Immunohistochemical staining helps in a definite diagnosis. The tumor cells are positive for SMA and vimentin, but are negative for pankeratin AE1/AE3 and low molecular weight cytokeratin. The extracellular matrix is positive for fibronectin. A wide resection of the tumor with continuous follow up is generally considered adequate.

\section{Conclusion}

On the basis of histopathological examination and immunohistochemical staining, a diagnosis of low grade myofibroblastic sarcoma of breast was established.

\section{References}

[1]. The Washington Manual of Surgical Pathology, $2^{\text {nd }}$ edition, Peter A. Humphrey, Louis P. Dehner, John D. Pfeifer, 749-755.

[2]. https:// diagnosticpathology.biomedcentral.com by Na-Hye Myong \& Jun-Won Min

[3]. https:// sagepub.com by Fernando Gonzalez - Palacois, Jose L. Enriquez, Pilar Miguel, Rosario Vazzquez, Monica Carcia-Cosio

[4]. Uncommon Malignancies - Journal of Clinical Oncology by P. B. Morgan. Sept. 1, 2005.

[5]. Am J Surg Pathol. 1998 Oct;22(10):1228-38. 